\title{
Galaxy Satellites and the Weak Equivalence Principle
}

\author{
Jose Ariel Keselman* \\ Physics department, Technion, Haifa 32000, Israel \\ Adi Nusser \\ Physics Department and the Asher Space Research Institute, Technion, Haifa 32000, Israel \\ P. J. E. Peebles \\ Joseph Henry Laboratories, Princeton University, Princeton, NJ 08544, USA
}

\begin{abstract}
Numerical simulations of the effect of a long-range scalar interaction (LRSI) acting only on nonbaryonic dark matter, with strength comparable to gravity, show patterns of disruption of satellites that can agree with what is seen in the Milky Way. This includes the symmetric Sagittarius stellar stream. The exception presented here to the Kesden and Kamionkowski demonstration that an LRSI tends to produce distinctly asymmetric streams follows if the LRSI is strong enough to separate the stars from the dark matter before tidal disruption of the stellar component, and if stars dominate the mass in the luminous part of the satellite. It requires that the Sgr galaxy now contains little dark matter, which may be consistent with the Sgr stellar velocity dispersion, for in the simulation the dispersion at pericenter exceeds virial. We present other examples of simulations in which a strong LRSI produces satellites with large mass-to-light ratio, as in Draco, or free streams of stars, which might be compared to "orphan" streams.
\end{abstract}

PACS numbers: 98.80.-k, 11.25.-w, 95.35.+d, 98.65.Dx 98.65.Fz, 98.10.+z, 98.56.Wm

\section{INTRODUCTION}

Kesden and Kamionkowski ([1, 2], hereafter KK06), in an independent application of the point made by Frieman and Gradwohl [3], analyzed an important astronomical test of the weak equivalence principle, that the acceleration of a freely moving test particle is independent of its composition, applied to nonbaryonic dark matter (DM). They pointed out that if the dark matter significantly violated the weak equivalence principle then the disruption of a satellite falling close to the host galaxy would tend to produce quite asymmetric stellar streams, a result of the non-inertial motion of the DM potential well that is holding the stars. This effect is contrary to the symmetric stellar streams of the Sagittarius (Sgr) satellite of the Milky Way (MW). The main purpose of this paper is to present a numerical simulation that demonstrates an exception to the Kesden-Kamionkowski effect.

Our exception, symmetric stellar streams, requires two conditions. First, the departure from the equivalence principle is large enough that it separates the stars and dark matter in a satellite falling toward its host galaxy before significant tidal disruption of the luminous parts of the satellite. Second, the mass in the central region of the satellite is dominated by stars. This second condition is consistent with the observation that the stars in the Sgr galaxy and stream have heavy element abundance patterns [4] that are considered to be characteristic of a more massive satellite such as the Large Magellanic Cloud [5], where the mass in the luminous central parts

*Electronic address: kari@tx.technion.ac.il is dominated by stars [6]. We show in the simulation in \II how the stellar system once separated from its dark matter halo suffers the usual pure gravitational tidal disruption as it falls toward the denser parts of the host, and can end up with a symmetric stellar galaxy and stream with properties reasonably similar to the Sgr system.

A significant departure from the weak equivalence principle in the dark sector would have other observable consequences. A simulation in $₫ \mathrm{IV}$ is meant to approximate the evolution of Draco, which seems to be dominated by DM, perhaps because it is strongly enough bound and has remained far enough from the galaxy to have avoided complete separation of the stars and DM. Other simulations in this section show the development of a coreless stellar stream, which might be related to the Orphan streams in the MW [7], and twin gravitationally bound cores, one dark and one stellar. The latter has not been observed but might prove to be interesting.

Our computation follows previous studies (reviewed in [8]) in expressing a departure from the weak equivalence principle as a fifth force of interaction in the dark sector. We take the interaction to be mediated by a scalar field that produces the fifth force, or LRSI,

$$
\mathbf{F}=-\frac{\beta G m^{2}}{r} e^{-r / r_{\mathrm{s}}}\left(\frac{1}{r}+\frac{1}{r_{\mathrm{s}}}\right) \hat{\mathbf{r}}
$$

between DM particles. Here $G$ is Newton's gravitational constant, $m$ is the mass of a DM particle, $\mathbf{r}$ is the separation vector, $\beta$ is a measure of the fifth force strength relative to gravity, and $r_{\mathrm{s}}$ is a "screening length". This fifth force applies only between DM particles; it adds to the usual gravitational interaction among DM and baryons. In the simulations presented here $r_{s}$ is much larger than 
the size of the galaxy so the fifth force is in effect an inverse square law. In the scalar field model for Eq. (1) the screening length scales with redshift as $r_{s} \propto(1+z)^{-1}[9]$. That is irrelevant for the effect on satellites of the MW but important in preventing any significant effect on the cosmological tests.

KK06 showed that the ratio of stellar masses in leading and trailing streams of a disrupting satellite can be quite sensitive to the value of $\beta$, and they argued that the approximate symmetry of the Sgr stellar streams implies $\beta<0.04$, in the notation of Eq. (1). Our alternative scenario for disruption follows if $\beta$ is on the order of unity, meaning the DM fifth force is about as strong as gravity on the scale of galaxies.

The next section outlines our numerical methods and parameter choices for building satellites of a MW-like galaxy and simulating the effects of gravity and an LRSI. The simulations use a version of the Gadget2 N-body code [10] modified to take account of the fifth force in Eq. (11). The numerical results for an Sgr-like satellite are presented in $\S$ III Because the picture requires that the Sgr galaxy contains little DM we pay particular attention to the departure of the stellar velocity dispersion from virial equilibrium as the satellite moves around the galaxy. We argue in $\S \nabla$ that this effect can reconcile the measured star velocity dispersion in the Sgr galaxy with a DM-free galaxy gravitationally bound by the mass in its stars. In $\S[\mathrm{IV}$ and $\mathrm{V}$ we comment on other issues to be explored in more detail.

\section{THE NUMERICAL MODELING}

We seek reasonably realistic simulations of the effect of an LRSI on observed satellites of the MW. In the simulations the galaxy is represented as a static potential produced by a dark matter halo, a stellar disk, and a stellar bulge. The behavior of the satellites is modeled by the N-body dynamics of two particle species, DM and stars, with the stellar component initially concentrated well within the DM potential, as suggested by [11], in initially spherical distributions. This description of initial conditions and numerical methods is supplemented by details in Appendix $\mathrm{A}$.

\section{A. Mass Distribution in the Host Galaxy}

The gravitational potential of our model for the MW is represented by the sum of three fixed components. The first is a spherical DM halo with potential

$$
\Phi_{\text {halo }}(r)=v_{\text {halo }}^{2} \ln \left(r^{2}+b^{2}\right),
$$

where $r$ is the distance from the center of the galaxy, $v_{\text {halo }}=131.5 \mathrm{~km} \mathrm{~s}^{-1}$, and $b=12 \mathrm{kpc}$. The second is a
Miyamoto-Nagai disk [12] with potential

$$
\Phi_{\text {disk }}=-\frac{G M_{\text {disk }}}{\sqrt{r_{\perp}^{2}+\left(a+\sqrt{z^{2}+d^{2}}\right)^{2}}}
$$

where $z$ and $r_{\perp}$ are cylindrical coordinates and $M_{\text {disk }}=$ $10^{11} \mathrm{M}_{\odot}, a=6.5 \mathrm{kpc}$, and $d=0.26 \mathrm{kpc}$. The third is a spherical Hernquist bulge [13] with potential

$$
\Phi_{\text {bulge }}=-\frac{G M_{\text {bulge }}}{r+c},
$$

where $M_{\text {bulge }}=3.4 \times 10^{10} \mathrm{M}_{\odot}$ and $c=0.7 \mathrm{kpc}$.

The DM particles feel the additional force given by the gradient of $\Phi_{\text {scalar }}=\beta \Phi_{\text {halo }}$. We neglect the exponential term in the scalar force in Eq. (1) because the size of the galaxy is supposed to be much smaller than the screening length $r_{\mathrm{s}}$.

\section{B. Construction of the satellites}

The initial DM and star distributions in a satellite are constructed to be spherically symmetric. The virial radius is $r_{\text {vir }}=r_{200} / \triangle$, where $r_{200}$ is the distance within which the mean mass density of DM plus stars is 200 times the present critical cosmological density (for Hubble parameter $H_{0}=70 \mathrm{~km} \mathrm{~s}^{-1} \mathrm{Mpc}^{-1}$ ), and $\triangle$ is discussed in the next subsection. The DM density profile at $r \leq r_{\text {vir }}$ is the Navarro, Frenk \& White form [14, 15],

$$
\rho_{\mathrm{DM}}(r)=\frac{\rho_{0}}{r / r_{\mathrm{vir}}\left(1+c_{\mathrm{v}} r / r_{\mathrm{vir}}\right)^{2}},
$$

where $c_{\mathrm{v}}$ is the concentration parameter and $\rho_{0}$ is a normalization factor. At $r>r_{\text {vir }}$ we choose an exponential DM density profile, as in [16],

$$
\rho_{\mathrm{DM}}(r)=\frac{\rho_{0}}{c_{\mathrm{v}}\left(1+c_{\mathrm{v}}\right)^{2}}\left(\frac{r}{r_{\mathrm{vir}}}\right)^{\alpha_{\mathrm{DM}}} \exp \left(-\frac{r-r_{\mathrm{vir}}}{r_{\mathrm{dec}}}\right) .
$$

The continuity of $\mathrm{d} \rho_{\mathrm{DM}} / \mathrm{d} r$ at $r=r_{\text {vir }}$ demands

$$
\alpha_{\mathrm{DM}}=\frac{-1-3 c_{\mathrm{v}}}{1+c_{\mathrm{v}}}+\frac{r_{\mathrm{vir}}}{r_{\mathrm{dec}}} \text {. }
$$

The undisturbed inner stellar component follows the modified Hubble density profile [17],

$$
\rho_{\mathrm{b}}(r)=\rho_{1}\left[1+\frac{r^{2}}{r_{\mathrm{c}}^{2}}\right]^{-2 / 3}, \text { at } r<r_{\mathrm{t}},
$$

where $r_{\mathrm{c}}$ is a core radius, $\rho_{1}$ is a normalization factor, and $r_{\mathrm{t}}$ is a truncation radius of the stellar component. At $r>r_{\mathrm{t}}$ the stellar density run is

$$
\rho_{\mathrm{b}}(r)=\rho_{1}\left(1+\frac{r_{\mathrm{t}}^{2}}{r_{\mathrm{c}}^{2}}\right)^{-3 / 2}\left(\frac{r}{r_{\mathrm{t}}}\right)^{\alpha_{\mathrm{b}}} \exp \left(-\frac{r-r_{\mathrm{t}}}{r_{\mathrm{dec}}}\right),
$$


where

$$
\alpha_{\mathrm{b}}=r_{\mathrm{t}}\left(\frac{1}{r_{\mathrm{dec}}}-\frac{3 r_{\mathrm{t}}}{{r_{\mathrm{c}}^{2}+r_{\mathrm{t}}^{2}}^{2}}\right)
$$

from continuity of $\mathrm{d} \rho_{\mathrm{b}} / \mathrm{d} r$ at $r=r_{\mathrm{t}}$.

We assign the satellite particles - DM and stars statistically isotropic velocity distributions that produce near dynamical equilibrium prior to significant perturbation by the host galaxy, using the variant of the method in [18] outlined in Appendix A Before placing the satellite in the gravitational potential of the host galaxy we compute the motions of the satellite particles for 20 gravitational dynamical times (as measured at the scale radius $r_{\text {vir }} / c_{\mathrm{v}}$ ) using the Gadget2 N-body code. The evolved density profiles are quite stable over this range of time.

\section{Parameter choices}

Throughout this study we set the ratio of the total DM to stellar mass within a satellite to be close to the global cosmic value [19]. The stellar mass fraction may be smaller than that in a satellite, as it is in larger galaxies (as reviewed in [17]), but that need not matter because excess DM in the outermost parts of the satellite could be stripped away before much happens to the stellar component.

We summarize in table \the parameters for the initial structures of the six model satellites we have chosen to illustrate possibly interesting effects of an LRSI. Entries 1 to 6 are the scale lengths in $\mathrm{kpc}$ and 7 and 8 are the concentration parameters defining the structure of the model satellites, as defined by Eqs. (5) to (10), prior to disturbance by the host.

The stellar component is embedded in a generally more extended DM component. The parameter $s_{\mathrm{b}} \equiv r_{\mathrm{c}} / r_{\mathrm{sc}}$ in entry 9 is the ratio of the stellar to $\mathrm{DM}$ core radii. According to [11], the range of values of this parameter in MW satellites is quite large, ranging from perhaps $s_{\mathrm{b}} \sim 0.05$ to close to unity. The strength of the LRSI relative to gravity is listed in entry 10 . The simulation S1 includes gravity alone $(\beta=0)$.

The host galaxy is centered on the origin of the coordinates, the disk is centered on the $Z=0$ plane, and the satellite moves in the YZ plane. The initial satellite position is in the plane of the disk at the distance (in $\mathrm{kpc}$ ), from the center of the galaxy, given in entry 11 . The initial velocity in entry 12 (in $\mathrm{km} \mathrm{s}^{-1}$ ) is in the positive $Z$ direction.

Entries 13 to 22 give the initial stellar and DM masses, in units of $10^{8} \mathrm{M}_{\odot}$, within various of the radii listed in entries 1 to 4 . The overdensity used to set the virial radius is $200 \times \triangle^{3}$, where $\triangle$ is listed in entry 23 . The exception to $\triangle=1$ is S3, where $\triangle=2.7$. This satellite is supposed to correspond to a high concentration $\left(c_{\mathrm{v}}=13.5\right)$ DM halo. However, the number of particles needed for proper numerical modeling is proportional to the fourth

\begin{tabular}{|l|c|c|c|c|c|c|}
\hline Simulation & $\mathrm{S} 1$ & $\mathrm{~S} 2$ & $\mathrm{~S} 3$ & $\mathrm{~S} 4$ & $\mathrm{~S} 5$ & $\mathrm{~S} 6$ \\
\hline \hline $1 . r_{\mathrm{c}}$ & 0.55 & 0.55 & 3.39 & 2.61 & 0.6 & 0.6 \\
$2 . r_{\mathrm{sc}}$ & 3.7 & 3.7 & 3.39 & 3.73 & 1.73 & 1.73 \\
$3 . r_{\mathrm{t}}$ & 1.67 & 1.67 & 10.17 & 10.4 & 1.81 & 1.81 \\
$4 . r_{\mathrm{vir}}$ & 18.65 & 18.65 & 17 & 18.6 & 8.65 & 8.65 \\
$5 . r_{\mathrm{dec}, \mathrm{bar}}$ & 0.1 & 0.1 & 0.1 & 0.1 & 0.1 & 0.1 \\
$6 . r_{\mathrm{dec}, \mathrm{dm}}$ & 0.1 & 0.1 & 0.1 & 0.1 & 0.1 & 0.1 \\
$7 . c_{\mathrm{v}, \mathrm{bar}}$ & 3 & 3 & 3 & 4 & 3 & 3 \\
$8 . c_{\mathrm{v}, \mathrm{dm}}$ & 5 & 5 & 5 & 5 & 5 & 5 \\
$9 . s_{\mathrm{b}}$ & 0.15 & 0.15 & 1 & 0.7 & 0.35 & 0.35 \\
$10 . \beta$ & 0 & 1 & 1 & 1 & 1 & 2 \\
$11 . Y_{(}(t=0)$ & 80 & 84.8 & 170 & 120 & 140 & 140 \\
$12 . v_{z}(t=0)$ & 80 & 80 & 140 & 200 & 280 & 280 \\
$13 . M_{\mathrm{dm}}(\infty)$ & 19 & 19 & 290 & 19.3 & 1.93 & 1.93 \\
$14 . M_{\mathrm{b}}(\infty)$ & 4.15 & 4.15 & 62.3 & 3.9 & 0.42 & 0.42 \\
$15 . M_{\mathrm{dm}}\left(r_{\mathrm{c}}\right)$ & 0.16 & 0.16 & 45 & 1.9 & 0.065 & 0.065 \\
$16 . M_{\mathrm{dm}}\left(r_{\mathrm{sc}}\right)$ & 3 & 3 & 45 & 3 & 0.31 & 0.31 \\
$17 . M_{\mathrm{dm}}\left(r_{\mathrm{t}}\right)$ & 0.94 & 0.94 & 150 & 9.3 & 0.33 & 0.33 \\
$18 . M_{\mathrm{dm}}\left(r_{\mathrm{vir}}\right)$ & 15 & 15 & 227 & 14.9 & 1.5 & 1.5 \\
$19 . M_{\mathrm{b}}\left(r_{\mathrm{c}}\right)$ & 0.6 & 0.6 & 9 & 0.48 & 0.061 & 0.061 \\
$20 . M_{\mathrm{b}}\left(r_{\mathrm{sc}}\right)$ & 4.15 & 4.15 & 9 & 0.93 & 0.29 & 0.29 \\
$21 . M_{\mathrm{b}}\left(r_{\mathrm{t}}\right)$ & 3 & 3 & 45 & 3 & 0.3 & 0.3 \\
$22 . M_{\mathrm{b}}\left(r_{\mathrm{vir}}\right)$ & 4.15 & 4.15 & 60 & 3.9 & 0.42 & 0.42 \\
$23 . \triangle_{24 .} N_{\mathrm{bar}}$ & 1 & 1 & 2.7 & 1 & 1 & 1 \\
$25 . N_{\mathrm{dm}}$ & 1.4 & 1.4 & 1.4 & 1.4 & 1.4 & 1.4 \\
$26 . \epsilon_{\mathrm{bar}}$ & 35 & 35 & 437 & 437 & 85 & 85 \\
$27 . \epsilon_{\mathrm{dm}}$ & 650 & 650 & 650 & 650 & 331 & 331 \\
\hline
\end{tabular}

TABLE I: Simulation parameters. See description in text.

power of the concentration (see Appendix $\mathrm{A}$ ). This becomes numerically expensive, and, as a compromise, we work with a larger $\triangle$ to imitate the desired larger concentration.

The parameters $N_{\text {bar }}$ and $N_{\mathrm{dm}}$ are the numbers of particles, in units of $10^{4}$, representing the mass distributions in stars and DM. The parameters $\epsilon_{\mathrm{bar}}$ and $\epsilon_{\mathrm{dm}}$ are the lengths in parsecs of the smoothing of the inverse square law for DM and stars in the numerical simulations, as defined in the Gadget2 code.

Six simulations for the initial conditions in Table \have been run with Gadget2. Only S1 does not include LRSI; it serves as a benchmark for comparison to the standard picture. Simulation S2 has initial conditions similar to S1 but is run with LRSI at the strength of gravity $(\beta=1)$. It is intended to produce the general properties of the Sgr galaxy and stream. The satellite S3 is constructed with $s_{\mathrm{b}}=1$, and has a DM-dominated core. It becomes almost completely dominated by DM as a result of an LRSI. It could be an analogue of Draco, though it is not intended to be a close match to any of its properties other than 


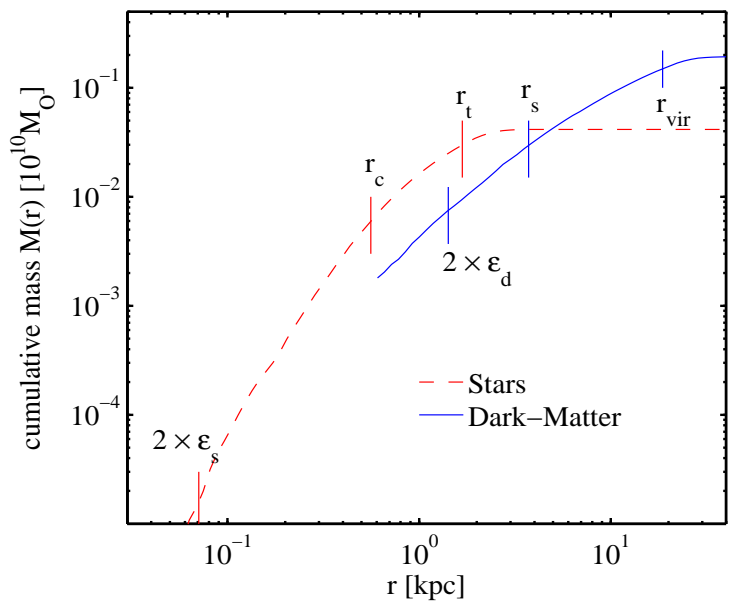

FIG. 1: The initial stellar and DM masses as functions of distance $r$ from the center of the satellites $\mathrm{S} 1$ and $\mathrm{S} 2$.

the high $M / L$ and the apparent absence of tidal streams. In S4 the stellar core is destroyed while the DM core survives, leaving a smooth stellar stream. Simulations S5 and S6 are attempts to illustrate the formation of twin cores, DM and stellar. They share the same initial conditions but differ by the LRSI strength $\beta$. For the Gadget 2 code parameters, we chose conservative values, resulting in conservation of at least $99.5 \%$ of the total energy for all simulations.

\section{MODELING THE SAGITTARIUS SYSTEM}

We describe here the evolution of model satellites S1 and S2 that are meant to simulate the formation of the Sgr galaxy and stream without and with the effect of a strong departure from the weak equivalence principle represented by an LRSI in the dark sector. We compare the models to observations in $\sqrt{\mathrm{V}}$

Initially the cores of S1 and S2 are dominated by the stellar mass out to $4 \mathrm{kpc}$ radius, as illustrated by the DM and stellar mass density runs in Fig. 1] As we have noted, and will discuss in more detail in $\$ \mathrm{~V}$, this is consistent with the fact that the Sgr stars have been compared to the population in the Large Magellanic Cloud (the LMC; e.g. [5]), in which the evidence is that the mass in the central region is dominated by stars $[6]$.

To get similar orbits we have assigned the same initial velocity but slightly different initial positions for S1 and S2 in the host galaxy (entries 11 and 12 in table I). Both simulations are run for $6 \mathrm{Gyr}$.

Evolutions of the particle distributions projected in the $Y Z$ plane perpendicular to the plane of the galaxy are shown in Figs. 2 and 3. The stellar core in S1 remains embedded in its DM halo as the satellite develops quite symmetric tidal streams. Early on the streams contain mainly DM, but after the first pericenter passage stars become prominent in the streams. The evolution of S2 is quite different, but it ends up with similar symmetric stellar streams. In a frame of reference moving with the DM core, the stellar core experiences an effective force that works against the gravitational pull of the DM core. At $\beta=1$ and at the initial position of S2 this effective force overcomes the gravitational pull of the DM core. The result is a nearly complete segregation of the stars and DM well before the first pericentric passage. The separated stellar core is then free to develop symmetric stellar tidal streams under purely gravitational dynamics. Meanwhile the DM core is completely disrupted.

The behavior of S2 is very different from the KK06 simulations. They focus on cases where the strength of the LRSI is weak enough that there is not segregation of the stellar and DM components prior to significant tidal disruption of the stellar system. In the KK06 simulations the effective force on the stars in the DM halo rest frame tends to pull stars out of one side of the halo, producing quite asymmetric stellar streams.

Fig. 4 shows measures of the evolution of S1 and S2. The vertical scales of each curve are arbitrarily normalized to fit the figure, independently in each panel. The dot-dashed curves are the satellite distance from the center of the host, showing pericenter passages. The solid blue curves show the rate of loss of the satellite stellar mass, and the dotted curves show the residual stellar mass. Here, the satellite is defined as the set of self-bound particles, which for S1 is calculated with both DM and stellar particles, and for S2 with stellar particles alone. As expected, the mass loss rate in both simulations is highest during pericenter passages where the tidal force is strongest. The figure also shows one-dimensional stellar velocity dispersions measured within $0.7 \mathrm{kpc}$ projected distance from the center of the satellite. The line-of-sight velocity dispersion for an observer at $(0,-8.5 \mathrm{kpc}, 0)$ relative to the center of the host galaxy is plotted as the thick black lines. The red dashed lines show the dispersions in the directions giving the maximum and minimum values. In these simulations the line-of-sight direction is close to the direction of maximum velocity dispersion at pericenter passages.

The satellite is constructed to be in dynamical equilibrium with an isotropic velocity distribution prior to disturbance by the host. The anisotropy that develops during pericenter passages, and the increase in the lineof-sight velocity dispersion, are combined results of the contamination of escaping particles and the transient departure from dynamic equilibrium, including the distortion of the satellite shape caused by the strong tidal force. The deviations from an isotropic stellar velocity distribution become larger in $\mathrm{S} 2$ once the stars are separated from the DM, the residual DM in S1 keeping the stars more tightly bound.

Fig. [5illustrates how transient disturbances to the stellar velocity distribution can affect dynamical mass estimates of the satellites S1 and S2. The blue curves show the evolution of the stellar mass and the stellar line-of- 


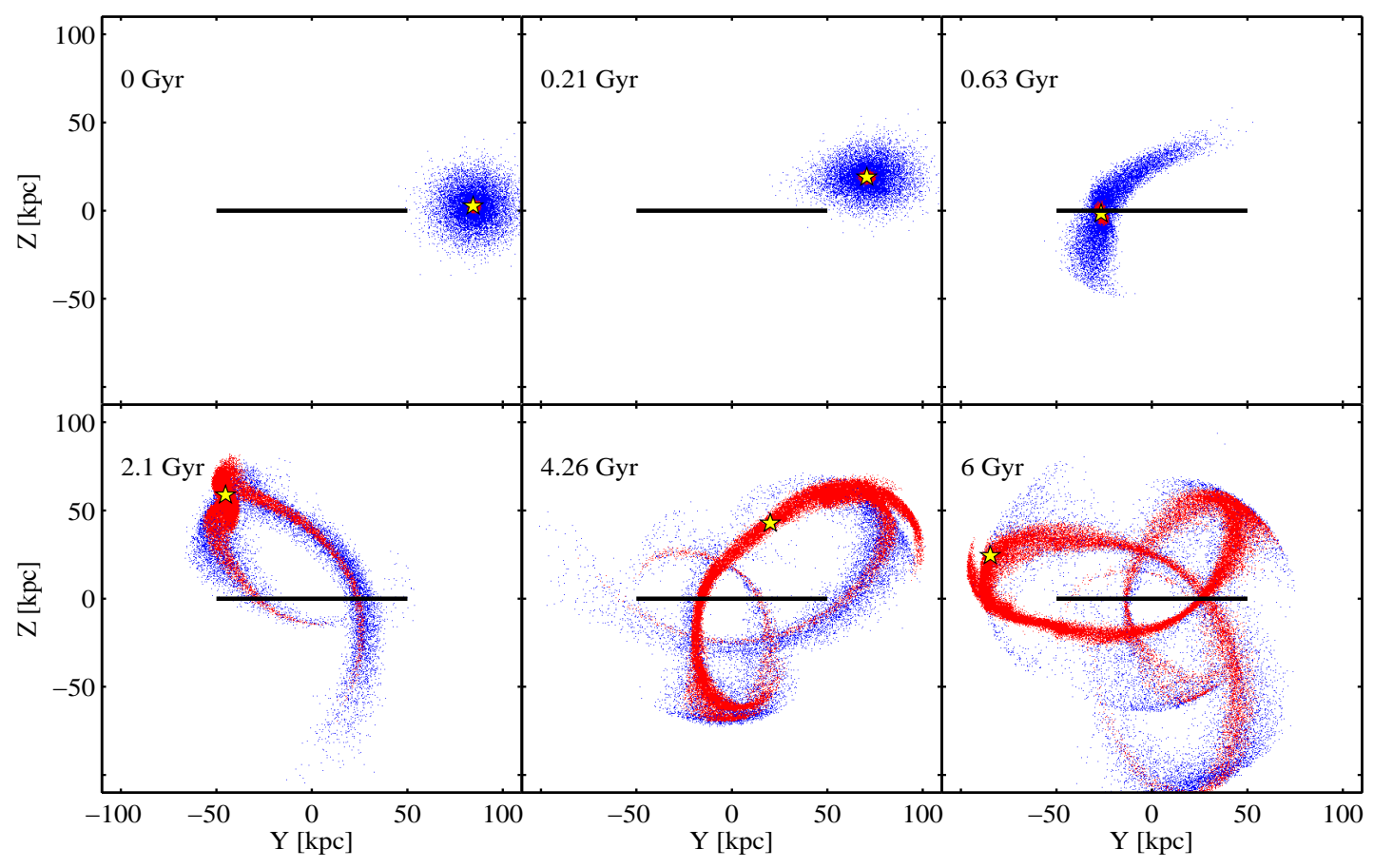

FIG. 2: Snapshots in the YZ plane of the evolution of the S1 satellite (without an LRSI). The stellar and DM particles are plotted as red open circles and blue dots, respectively. For clarity, only a subset of the stellar particles are plotted. The yellow star indicates the location of the most bound stellar particle.

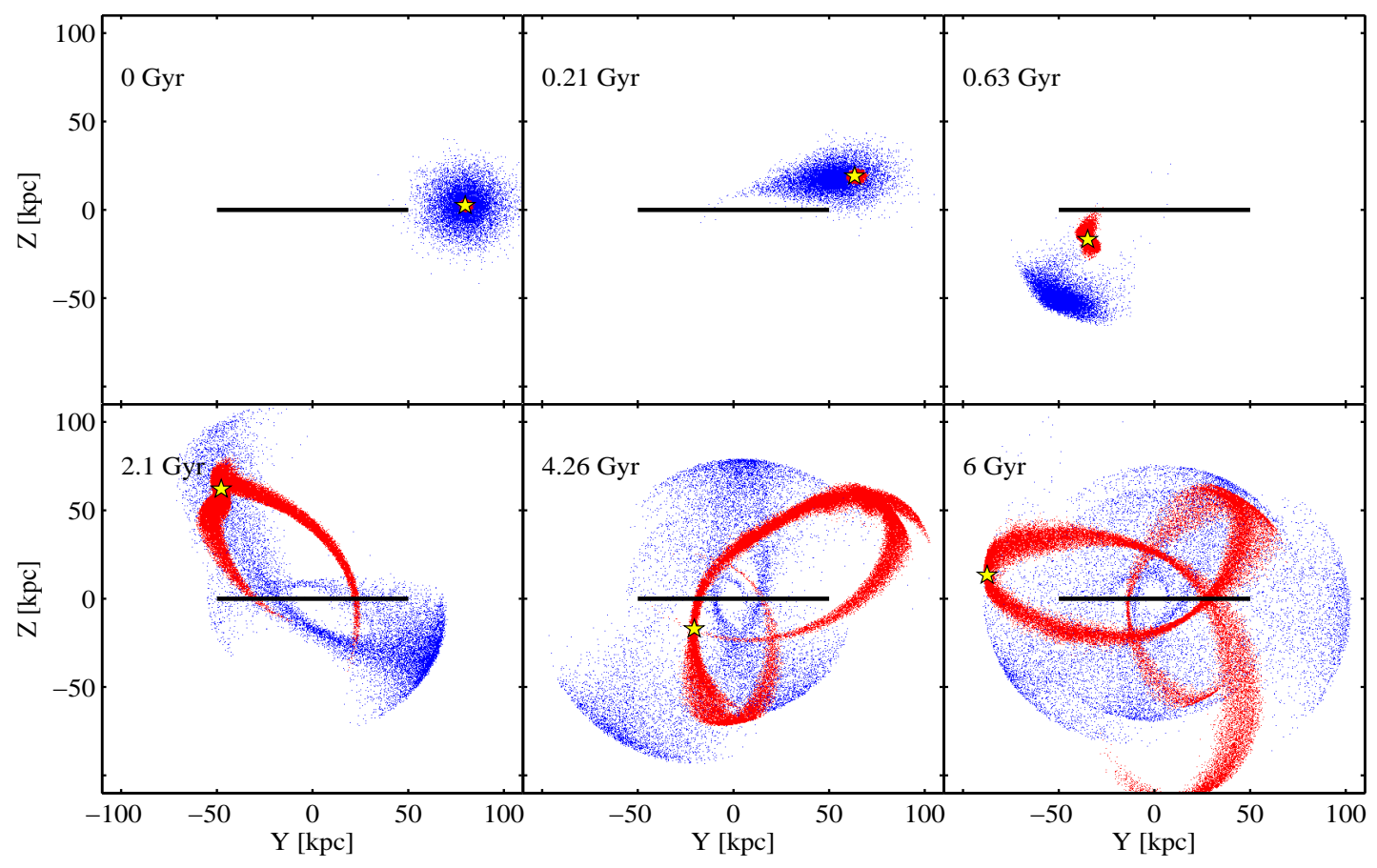

FIG. 3: The same as Fig. 2 but for S2 with an LRSI with $\beta=1$. 


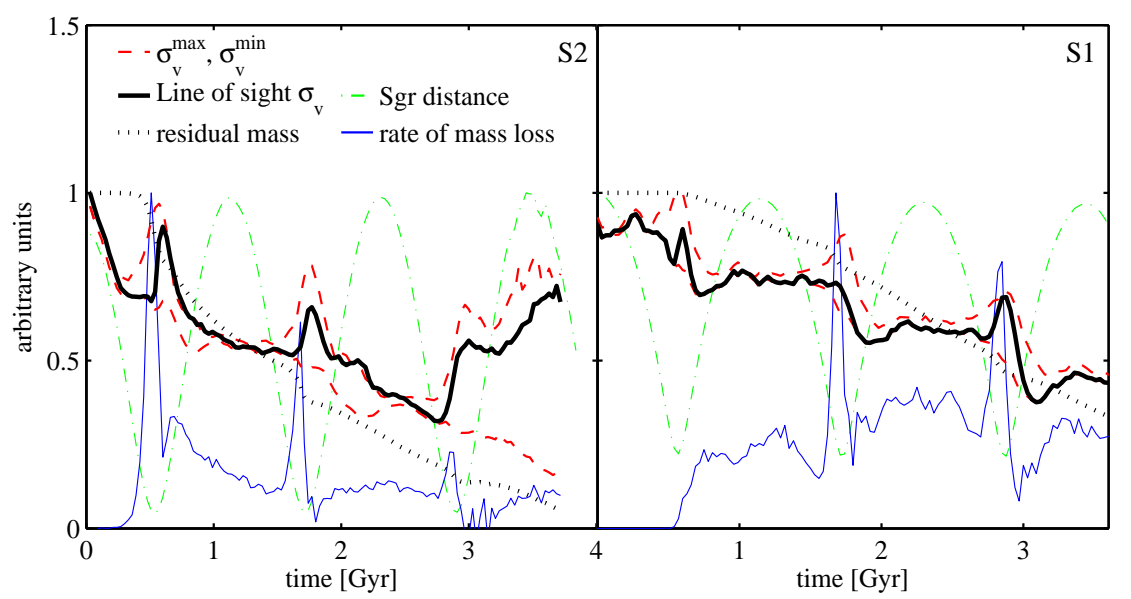

FIG. 4: Measures of evolution of the S1 (panel to the right) and S2 (to the left) simulations.

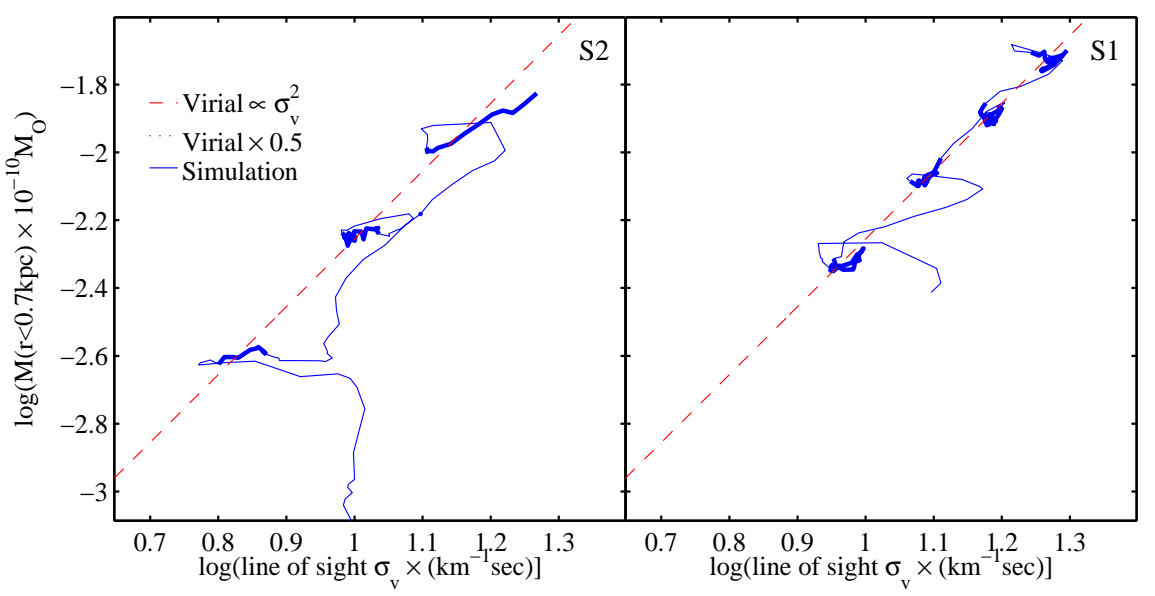

FIG. 5: Evolution of the satellite bound stellar mass and stellar line-of-sight velocity dispersion follows the blue lines commencing near the top right corner of each panel and ending toward the lower left. At the thick portions the velocity anisotropy is less than $15 \%$. The dashed line varies as ${\sigma_{\mathrm{v}}}^{2}$, as expected for dynamical equilibrium in a satellite with fixed size.

sight velocity dispersion $\sigma_{\mathrm{v}}$, both computed within projected radius $0.7 \mathrm{kpc}$ perpendicular to the line of sight. The thick portions of the curves correspond to times when ${\sigma_{\mathrm{v}}}^{\text {max }}-{\sigma_{\mathrm{v}}}^{\text {min }}<0.15 \sigma_{\mathrm{v}}{ }^{\text {mean }}$, where $\sigma^{\text {mean }}$ is the RMS value averaged over all directions. The satellites start at the top-right corners and end toward the lowerleft after 4.17 Gyr for S1 and 3.3 Gyr for S2. The dashed red lines show how the velocity dispersion $\sigma_{\mathrm{v}}$ would vary with the mass, $M(<r) \propto \sigma_{\mathrm{v}}{ }^{2}$, at dynamical equilibrium and fixed radius $r$. The blue curves pass close to this condition when the velocity anisotropy is small. At the thin portions of the line the satellite is closest to the host galaxy and most disturbed. Here the mass is smaller than expected from the velocity dispersion $\sigma_{\mathrm{v}}$ under the assumption of dynamical equilibrium, by a factor as large as two and even larger for S2 towards the final time. Earlier studies of the possible importance of tidally-driven departures from dynamical equilibrium differ, but some indicate that velocity dispersions of longlived dwarf satellites can be interpreted as the effect of a departure from equilibrium rather than the presence of dark matter [20, 21, 22, 23].

Fig. 6] shows that the stellar velocity dispersions in the cores of S1 and S2 are close to constant across the face of the galaxy. The curves at $t=3 \mathrm{Gyr}$ are higher than at $t=2.8 \mathrm{Gyr}$ in both $\mathrm{S} 1$ and $\mathrm{S} 2$, because the former coincides with a pericentric passage in both simulations.

The evolution of the mean surface density profile as a function of projected distance $R$ from the center of the satellite is shown in Fig. 7. The stellar component plotted as the thin lines preserves a flat density in the inner regions, in agreement with [24]

The parameter KK06 use as a measure of the degree of symmetry of the tidal stream is the ratio of the number of 


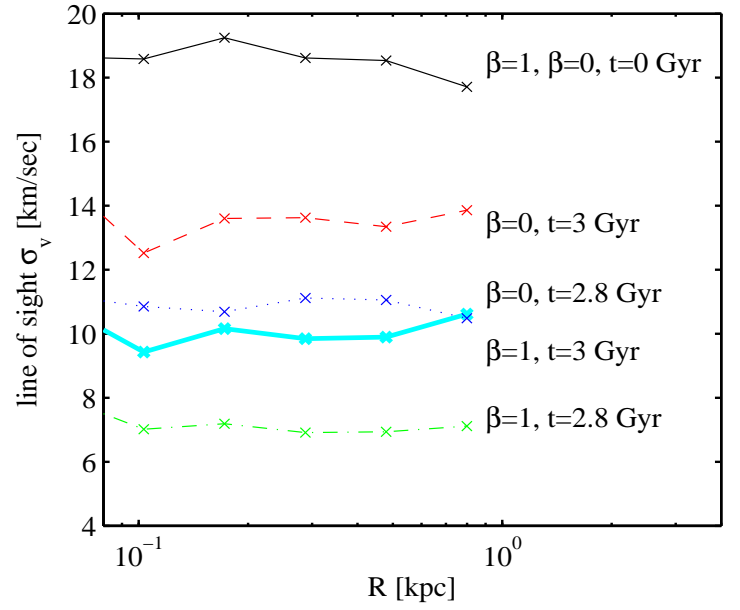

FIG. 6: Line-of-sight stellar velocity dispersions as functions of projected distance from the satellite center are shown for $\mathrm{S} 1(\beta=0)$ and $\mathrm{S} 2(\beta=1)$ at various times indicated in the figure.

leading to trailing stars. KK06 find that in all their simulations without an LRSI this ratio is never lower than 0.5, while in the simulations with $0.04 \leq \beta \leq 0.09$ it never exceeds 0.2 . Indeed, this supports the conclusion that a weak LRSI is seriously constrained by the observations of Sgr. In the strong LRSI case we are considering, there is some asymmetry in the early stages of evolution, which begins to decay right after the DM leaves the stellar core. At $t=1.7$ Gyr the ratio of the number of leading to trailing stars, excluding particles that are closer than $4 \mathrm{kpc}$ to the center, is 1.09 and 0.817 for $\mathrm{S} 1$ and $\mathrm{S} 2$ respectively. At $t=2.85 \mathrm{Gyr}$ the ratios are 0.96 and 0.88 .

We discuss how these models for the Sgr galaxy and stream compare to the observations in $\mathrm{gV}$, after presenting simulations of a few other situations of possible astronomical interest.

\section{OTHER MANIFESTATIONS OF AN LRSI}

The mass-to-light ratio of the Draco satellite, at distance $D \approx 80 \mathrm{kpc}$ from the MW, is estimated to be $M / L>100$ Solar units [25]. This large value is not reasonably explained by deviations from equilibrium [26] such as those occurring near pericenter passages discussed in the previous section; it more likely means the mass is dominated by DM throughout this satellite. In the standard scenario, tidal mass loss may result in an increased $M / L$ ratio [24], however this effect is not strong enough to explain highly DM dominated satellites as Draco. LRSI offers a mechanism that can work either separately or in tandem with the usual postulate that photoionization heating by the UV background has suppressed star formation [27, 28, 29], provided the LRSI is strong enough to have stripped away most of the stars but weak enough to leave the most tightly bound stars in a DM halo that remains bound.

Our simulation S3, with $\beta=1$, illustrates a case of significant but not complete loss of stars in the LRSI picture. The stellar and DM core radii initially are the same $\left(s_{\mathrm{b}}=1\right.$ in table $\left.\mathbb{I}\right)$, and the central region is dominated by DM (at close to the cosmic baryonic to DM mass ratio). One sees in Fig. 8 that the LRSI pulls most of the stellar particles out of the DM halo early in the evolution, in the direction opposite to the motion. That leaves a bound DM halo with a tighter concentration of stars, in which the ratio of bound DM mass to stellar mass is 16 times larger than the initial value.

Simulation S4 illustrates the development of the coreless stellar stream in Fig. 9 that might be compared to the orphan streams in the MW. In this simulation the stellar core is not gravitationally self-bound (the satellite core mass is not dominated by stars) so it is disrupted by the segregation driven by the strong LRSI. Even after $7.4 \mathrm{Gyr}$ the stars remain in a smooth stream, little disturbed by the small mass left in the DM core in this simulation.

Simulations S5 and S6 are tuned to explore the possibility that both the DM and stellar cores survive separation by an LRSI, ending up as separate self-bound objects. Since a DM core is bound by the combined effect of gravity and the LRSI its binding energy is enhanced by the factor $1+\beta$ relative to a stellar system with the same mass distribution. This means that, once we arrange for a self-bound remnant stellar core, a DM core will survive if $\beta$ is large enough. We did not find bound twin cores in S5 with $\beta=1$. Fig. 10, for $\beta=2$ (S6), shows twin cores, but the remnant bound DM halo mass is only $1 \%$ the mass of the original satellite.

\section{DISCUSSION}

The central point of this paper is that a strong departure from the weak equivalence principle for nonbaryonic dark matter, with $\beta \simeq 1$ in the LRSI model in Eq. (11), can produce a system that resembles the Sgr galaxy and stream from a progenitor that seems reasonable from the point of view of the astronomy. We have also taken note of other modes of satellite disruption that might be observationally interesting.

It is important for our picture of the origin of the Sgr system that the mass distribution in the central part of the Sgr progenitor is dominated by stars. The thought that this might be so is motivated by the observation that stellar element abundances in the Sgr galaxy and stream have been compared to LMC stars (e.g. [5]), and the evidence that the mass in the luminous central parts of the LMC is dominated by stars [6].

We have aimed for a progenitor in our simulation that has structure similar to the LMC (though a rough similarity is likely all that could be meaningful). Our S1 and $\mathrm{S} 2$ cases each have initial total mass $\approx 2.2 \times 10^{9} \mathrm{M}_{\odot}$. 


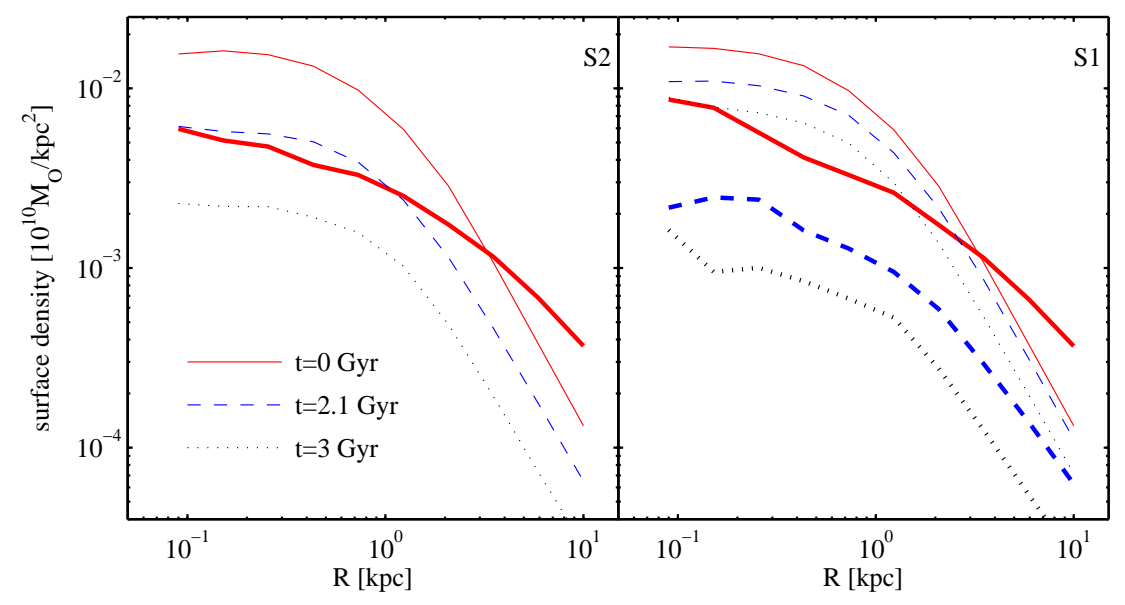

FIG. 7: The mean stellar surface density profiles at different times for S1 (right panel) and S2 (to the left). The DM profiles for $\mathrm{S} 1$ are plotted as the thicker lines.

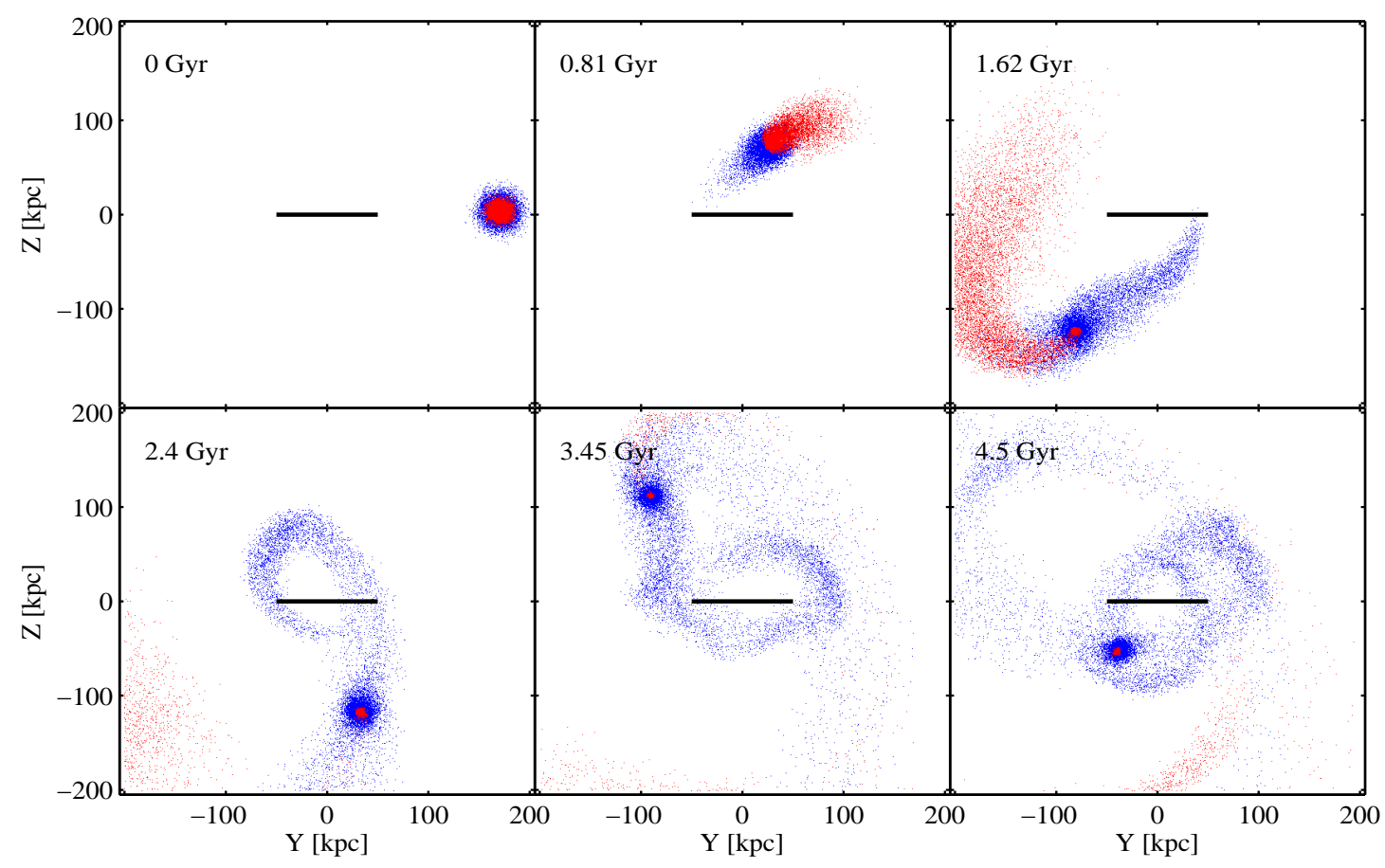

FIG. 8: Illustration S3 of the development of a satellite that retains a small but nonzero fraction of its stars. The orientation is the same as in Figs. 2 and 3 Red open circles are the stellar component, blue dots the DM.

The virial radius is slightly less than $20 \mathrm{kpc}$, and stars dominate the mass within $4 \mathrm{kpc}$ radius. Kim et al. [6] find that the LMC mass is $\approx 3.5 \times 10^{9} \mathrm{M}_{\odot}$ within $4 \mathrm{kpc}$ radius, and the total disk mass is $2.5 \times 10^{9} \mathrm{M}_{\odot}$ within $7.3 \mathrm{kpc}$ radius. By these measures the initial structures of S1 and S2 are reasonably similar to the LMC.

In S2, with the strong LRSI, the bound mass in stars at the third pericenter passage at $3 \mathrm{Gyr}$ is $5.2 \times 10^{7} \mathrm{M}_{\odot}$. At stellar mass-to-light ratio $M / L=2$ Solar units the luminosity would be $L \sim 1 \times 10^{8} L_{\odot}$. This is fairly close to the measured luminosity of the Sgr galaxy, in the range $(2-5.8) \times 10^{7} L_{\odot}[30,31]$.

In our model the Sgr galaxy no longer contains dark matter. Estimates of $M / L$ for this galaxy are larger than 


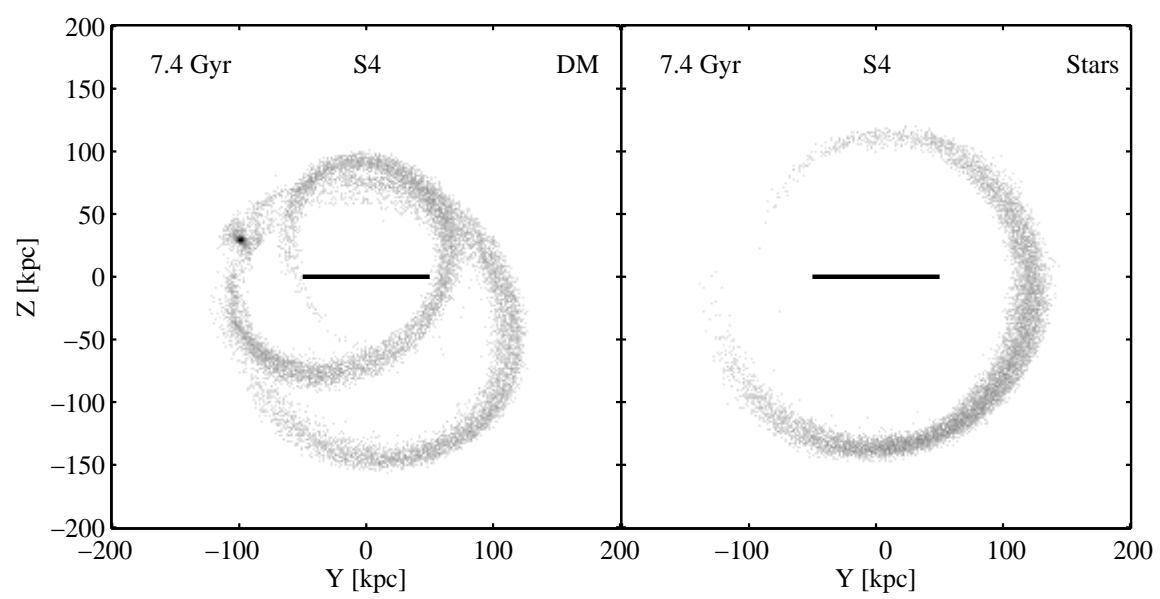

FIG. 9: Mass distributions near the end of simulation S4.

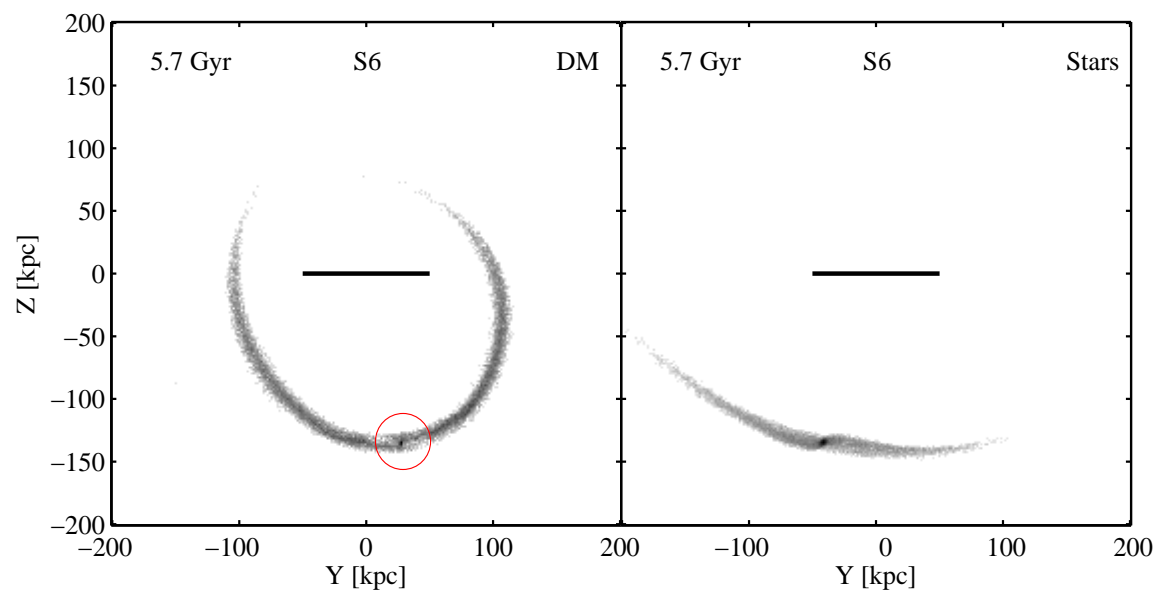

FIG. 10: Simulation S6. Circled in red is the remnant DM core.

expected for stars; thus from a recent program of observations 32] finds $M / L=17.5$. This assumes dynamical equilibrium, however, and we have illustrated in Figs. 4 and 5 how the galaxy may be disturbed from equilibrium by its host. In $\mathrm{S} 2$ the satellite at $3 \mathrm{Gyr}$ is at pericenter $18 \mathrm{kpc}$ from the center of the host galaxy, similar to the present distance of the Sgr galaxy, $16 \mathrm{kpc}$ [33]. Also, although our host galaxy is rigid its mass distribution is designed to resemble that of the MW. At this third pericenter passage the line-of-sight velocity dispersion within the innermost $0.7 \mathrm{kpc}$ of the simulated satellite is close to constant across the core at $\sigma=9.8 \mathrm{~km} \mathrm{~s}^{-1}$ (Fig. 6). This is in line with the finding of [32] that the velocity dispersion in the Sgr galaxy is close to constant across the inner $0.7 \mathrm{kpc}$ from the center defined by M54 at $\sigma=9.6 \mathrm{~km} \mathrm{~s}^{-1}$.

The departure from dynamical equilibrium at pericenter, and the large apparent value of the mass, also occurs in conventional gravity without the LRSI, as in S1, and in the simulations by [34, 35]. The effect is larger in S2 than S1, however, because of the greater fragility of the stellar core after the early loss of the DM.

Our conclusion from these comparisons of S2 to the observations is that it is possible to obtain a reasonably realistic picture of the origin of the Sgr system, including a DM-free galaxy and a symmetric stream, under the effect of a strong departure from the weak equivalence principle in the dark matter. It would be interesting to study the effects of LRSI on the features of the tidal streams, as done in [36].

Our simulations suggest alternative interpretations of other observations. The example in Fig. 8, a satellite that loses most but not all of its stars, might be compared to Draco. A rough analytic argument that the Draco DM halo can retain its most tightly bound stars against the effect of a strong LRSI goes follows. If the MW rotation curve for baryons is close to flat at $v_{c}=220 \mathrm{~km} \mathrm{~s}^{-1}$ at distance $D$ then a star in the DM halo rest frame experiences acceleration $\beta v_{c}^{2} / D$. The gravitational accelera- 
tion produced by the mass in the satellite at distance $r$ from its center is maximum at $G M(<r) / r^{2} \simeq 2 \sigma^{2} / r_{c}$ at the core radius $r_{c}$. According to 25] the stellar core radius of Draco is $160 \mathrm{pc}$. These numbers indicate that the one-dimensional stellar velocity dispersion must be $\sigma=7 \beta^{1 / 2} \mathrm{~km} \mathrm{~s}^{-1}$ to keep stars in the core of Draco at its present distance $D$. Since the measured dispersion is in the range $\sigma=8.5-10.7 \mathrm{~km} \mathrm{~s}^{-1}$ [25], we conclude that a strong LRSI, $\beta \sim 1$, allows Draco to contain stars as long as it has not ventured much closer to the MW than its present distance.

In the numerical simulation in Fig. 8 there are remnant bound stars, the ratio of bound DM to stellar mass is an order of magnitude larger than the original value with the DM remaining dominant at all radii, and the stars that have escaped are broadly scattered. This is in line with the properties of Draco. Whether more detailed measures of the simulated remnant satellite can be consistent with what is observed in Draco is a subject for separate study.

Another example for closer study is the difference between the observed numbers of satellites and the far greater numbers of DM halos predicted by the $\Lambda \mathrm{CDM}$ cosmology 37, 38, 39]. This usually is taken to mean that many DM halos are not visible because they were unable to accrete or retain photoionization-heated plasma [27, 28, 29]. The possibility offered by an LRSI is that many halos are not visible because stars have been removed from their DM halo, perhaps largely, as may have happened to Draco, or entirely.

Depending on the structure of the progenitor, stars removed from the DM may be broadly dispersed or may end up in a coreless stream, as in Fig. 9. The latter offers an interpretation the orphan streams in the MW (e.g. 7]). Orphan streams can form by tidal disruption in standard gravity acting on satellites in pericentric passages close enough or repeated often enough to make it difficult to identify the remnant satellite or totally disrupt it. In this scenario the formation time of coreless streams could be quite long [23]. In the LRSI scenario demonstrated in Fig. 9 coreless streams may form rapidly, and could be found in orbits with large pericentric distances. This behavior might be detectable, and is a signature of a strong LRSI.

Some stars separated from the DM may end up in gravitationally bound DM-free dwarf galaxies. There are discussions of dwarfs with mass-to-light ratios that might be considered characteristic of a normal stellar population [40]. DM-free dwarfs would be an interesting challenge to the standard theory. If the stars in the progenitor were much more strongly concentrated than the DM then a normal gravitational tide could strip away most of the DM, leaving a bound system dominated by stars, but it would be a delicate operation. An LRSI offers an alternative that operates in a straightforward way.

A recent study [41] challenges the model presented here. The main concern is that the satellite S2 is tightly constrained in the initial distance in which it may first enter the MW, since it is too light for dynamical friction to modify its orbit into the observable one. It is important to notice that in the standard model (without LRSI), Sgr could have many different possible orbital histories which lead to its currently observed setup. According to [42], in one family of solutions the Sgr may have entered the MW at a galactocentric distance close to its current apocentre of $60 \mathrm{kpc}$, with a mass of $10^{9} \mathrm{M}_{\odot}$. In this model, the Sgr is indeed too light for gravitational friction to have any significant effect on its orbit. This model is consistent with our satellite model S2. In another distinct family of solutions, the Sgr could enter the MW at a distance of more than $200 \mathrm{kpc}$ with a total mass of about $10^{11} \mathrm{M}_{\odot}$ which is 100 times more massive than S2. In this scenario, dynamical friction plays a key role in bringing Sgr to its current orbit, while along the way it looses more than $99 \%$ of its mass. This scenario is consistent with [43].

We have run a simulation of this 'heavy' Sgr scenario, including a 'live' MW halo to account for dynamical friction. The results are illustrated in Fig. 11. Here, the MW halo is modeled with an NFW profile of concentration 15 , mass of $2 \times 10^{12} \mathrm{M}_{\odot}$, and virial radius $250 \mathrm{kpc}$. The number of particles is $2 \times 10^{4}$, and the smoothing $8 \mathrm{kpc}$. The Galactic disk and bulge have the same parameters as in the previous simulations, however the bulge is not a static potential, but a single massive particle smoothed at $9 \mathrm{kpc}$ with a spline kernel, as all particles in Gadget2, and the center of the stellar disk static potential follows the particle that represents the bulge. The initial Sgr has a baryonic component of mass $4 \times 10^{10} \mathrm{M}_{\odot}$ with a truncation radius of $3.6 \mathrm{kpc}$ and is represented by $7 \times 10^{4}$ particles, smoothed at $55 \mathrm{pc}$. The DM component is five times more massive, has a virial radius of $29 \mathrm{kpc}$ represented with $2 \times 10^{4}$ particles, and smoothed at $850 \mathrm{pc}$. Initially, the $\mathrm{Sgr}$ is positioned in the stellar-disk plane at a GC distance equal to the Galactic virial radius of $250 \mathrm{kpc}$, and its velocity is set to the baryonic circular velocity of $185 \mathrm{kms}^{-1}$, perpendicular to the stellar-disk plane.

At the initial position in this simulation the mass and concentration of the Sgr DM halo are large enough to prevent separation between stars and DM. This is important, because while the baryons are bound to the DM core, the orbital dynamics is almost independent of $\beta$, since changing $\beta$ is equivalent to a change in the gravitational constant $G$, which in turn is equivalent to a transformation in the time variable $t \rightarrow t \times \sqrt{\beta+1}$. Hence, the Sgr begins to sink in the usual way, while experiencing dynamical friction and loosing mass to the MW. Only when the Sgr is in an orbit similar to the one which is inferred directly from the observations (i.e. with an apocenter of $60 \mathrm{kpc}$ ), the DM halo becomes small enough to allow full separation. Afterwards the Sgr develops symmetric tidal arms, in a similar way to $\mathrm{S} 1$ and $\mathrm{S} 2$, and because it looses about $99 \%$ of its mass, it is similar to the Sgr today. This works also for larger turn-around distances, since as mentioned above, the dynamics are independent of $\beta$ as long as the DM and stellar cores are 
initially tightly bound.

\section{CONCLUDING REMARKS}

We have addressed some of the issues in astronomy that are raised by the postulate of a strong departure from the weak equivalence principle by nonbaryonic dark matter. One must also consider the effect on structure formation on larger scales. A preliminary analysis [44] of the effect of adding a strong LRSI to the $\Lambda$ CDM cosmology indicates an improved fit of theory to observations of voids, which are more completely emptied by the LRSI, and of giant galaxies, which complete major mergers sooner. Since the DM clusters more rapidly in the LRSI scenario, the baryons on scales below $r_{\mathrm{sc}}$ would be expected to lag behind the DM. This effect could leave a fraction of the baryons out of galactic haloes and help explain the missing baryon problem reviewed in [45]. We plan to present a more detailed and systematic analysis of these considerations using the numerical methods developed here with the further adjustment of the numerical code to take account of the departure from an LRSI inverse square law in Eq. (11).

As we noted in the last section there are still other issues to consider. One is whether a satellite with all the properties of Draco may naturally form in the strong LRSI picture. Another is the excess satellite problem [37, 38, 39]. We stress here that LRSIs do not necessarily imply DM free satellites. In fact, small but high concentrated DM halos could easily trap the stellar components so that full segragation never happens. This means that LRSI may even help increase the M/L ratio by yanking only the least bound stars. An interesting quantitative test in a pure particle simulation of the formation of an $L_{*}$ galaxy would count satellites that are entirely stripped of the particles that represent baryons, satellites that retain enough stellar particles to be visible, and satellites that fall in at low redshift and are capable of producing symmetric and orphan stellar streams.

We are considering a substantial departure from standard physics, but it is in the little explored dark sector where our present physics is so very simple as to seem possibly suspicious. Thus we feel that explorations of more complicated dark sector physics, while speculative, are worthwhile. Our studies of the observational tests of the speculative idea that there is a long-range departure from the weak equivalence principle in the dark sector have not proved to be discouraging so far.

\section{Acknowledgment}

We have benefited from instructions on the properties of the Sgr system by Kathryn Johnston and Gerry Gilmore and from discussions of numerical simulations of satellite galaxies with Mike Kesden and Glennys Farrar. This research is supported in part in Israel by the German-Israeli Foundation for Scientific Research and Development, by the German-Israeli Foundation for Research and Development, by the Asher Space Research Fund, by the Israel Science Foundation (grant No. 203/09), by the Asher Space Research Institute, and by the Winnipeg Research Fund, and in part in the USA by Princeton University. We also acknowledge two anonymous referees whose comments helped improve this text.
[1] M. Kesden and M. Kamionkowski, Phys. Rev. D 74, 083007 (2006), arXiv:astro-ph/0608095.

[2] M. Kesden and M. Kamionkowski, Phys. Rev. Lett. 97, 131303 (2006), arXiv:astro-ph/0606566.

[3] J. A. Frieman and B.-A. Gradwohl, Science 260, 1441 (1993).

[4] L. Sbordone, P. Bonifacio, R. Buonanno, G. Marconi, L. Monaco, and S. Zaggia, A\&A 465, 815 (2007), arXiv:astro-ph/0612125.

[5] R. Carrera, C. Gallart, E. Hardy, A. Aparicio, and R. Zinn, AJ 135, 836 (2008), 0710.3076.

[6] S. Kim, L. Staveley-Smith, M. A. Dopita, K. C. Freeman, R. J. Sault, M. J. Kesteven, and D. McConnell, ApJ 503, 674 (1998).

[7] V. Belokurov, N. W. Evans, M. J. Irwin, D. Lynden-Bell, B. Yanny, S. Vidrih, G. Gilmore, G. Seabroke, D. B. Zucker, M. I. Wilkinson, et al., ApJ 658, 337 (2007), arXiv:astro-ph/0605705.

[8] G. R. Farrar and P. J. E. Peebles, ApJ 604, 1 (2004), arXiv:astro-ph/0307316.

[9] S. S. Gubser and P. J. E. Peebles, Phys. Rev. D 70, 123510 (2004), arXiv:hep-th/0402225.
[10] V. Springel, MNRAS 364, 1105 (2005), arXiv:astro$\mathrm{ph} / 0505010$.

[11] J. Peñarrubia, A. W. McConnachie, and J. F. Navarro, ApJ 672, 904 (2008), arXiv:astro-ph/0701780.

[12] M. Miyamoto and R. Nagai, PASJ 27, 533 (1975).

[13] L. Hernquist, ApJ 356, 359 (1990).

[14] J. F. Navarro, C. S. Frenk, and S. D. M. White, ApJ 462, 563 (1996), arXiv:astro-ph/9508025.

[15] J. F. Navarro, C. S. Frenk, and S. D. M. White, ApJ 490, 493 (1997), arXiv:astro-ph/9611107.

[16] V. Springel and S. D. M. White, MNRAS 307, 162 (1999).

[17] J. Binney and S. Tremaine, Galactic Dynamics: Second Edition (Galactic Dynamics: Second Edition, by James Binney and Scott Tremaine. ISBN 978-0-691-13026-2 (HB). Published by Princeton University Press, Princeton, NJ USA, 2008., 2008).

[18] S. Kazantzidis, J. Magorrian, and B. Moore, ApJ 601, 37 (2004), arXiv:astro-ph/0309517.

[19] J. Dunkley, E. Komatsu, M. R. Nolta, D. N. Spergel, D. Larson, G. Hinshaw, L. Page, C. L. Bennett, B. Gold, N. Jarosik, et al., ArXiv e-prints (2008), 0803.0586. 

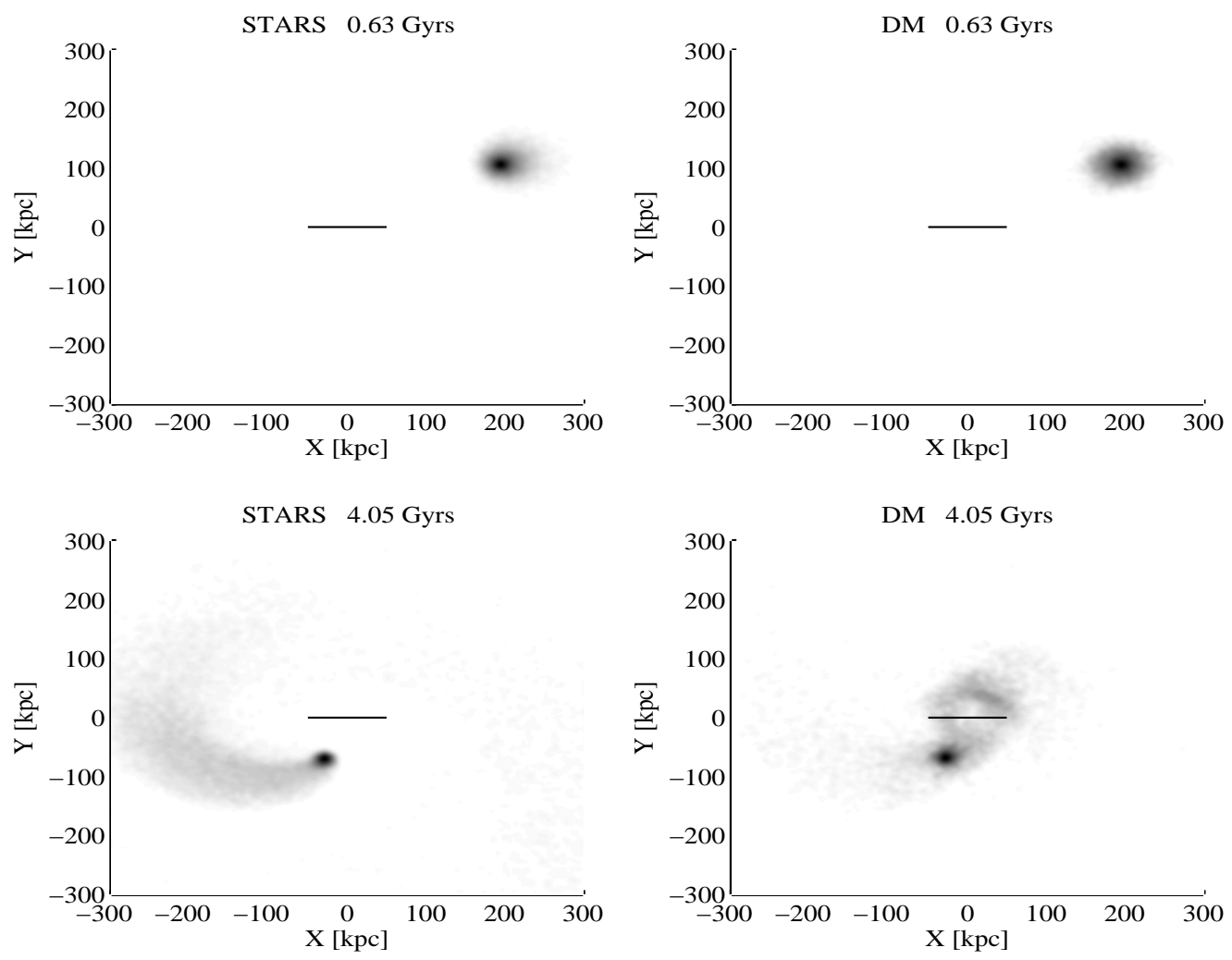

STARS 8.04 Gyrs (ZOOMED)
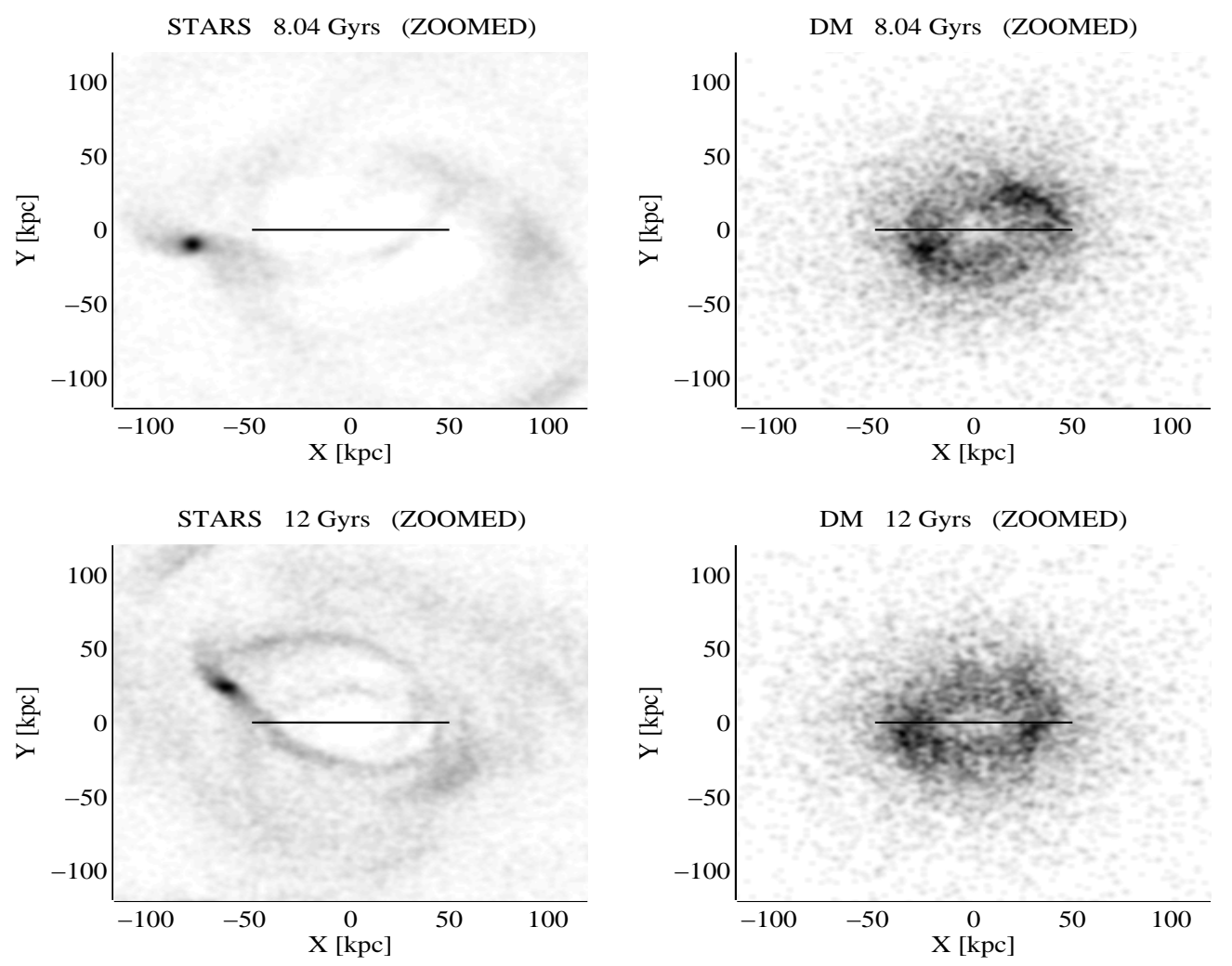

FIG. 11: Mass distribution for stars and DM in the 'heavy' Sgr simulation. 
[20] K. S. Oh, D. N. C. Lin, and S. J. Aarseth, ApJ 442, 142 (1995).

[21] S. Piatek and C. Pryor, AJ 109, 1071 (1995).

[22] P. Kroupa, New Astronomy 2, 139 (1997).

[23] R. S. Klessen and P. Kroupa, ApJ 498, 143 (1998), arXiv:astro-ph/9711350.

[24] J. Peñarrubia, J. F. Navarro, and A. W. McConnachie, ApJ 673, 226 (2008), 0708.3087.

[25] M. Odenkirchen, E. K. Grebel, D. Harbeck, W. Dehnen, H.-W. Rix, H. J. Newberg, B. Yanny, J. Holtzman, J. Brinkmann, B. Chen, et al., AJ 122, 2538 (2001), arXiv:astro-ph/0108100.

[26] R. S. Klessen and H. Zhao, ApJ 566, 838 (2002), arXiv:astro-ph/0110427.

[27] A. J. Benson, C. G. Lacey, C. M. Baugh, S. Cole, and C. S. Frenk, MNRAS 333, 156 (2002), arXiv:astro$\mathrm{ph} / 0108217$.

[28] A. J. Benson, C. S. Frenk, C. G. Lacey, C. M. Baugh, and S. Cole, MNRAS 333, 177 (2002), arXiv:astro$\mathrm{ph} / 0108218$.

[29] A. J. Benson, C. S. Frenk, C. M. Baugh, S. Cole, and C. G. Lacey, MNRAS 343, 679 (2003), arXiv:astro$\mathrm{ph} / 0210354$.

[30] R. A. Ibata, R. F. G. Wyse, G. Gilmore, M. J. Irwin, and N. B. Suntzeff, AJ 113, 634 (1997), arXiv:astro$\mathrm{ph} / 9612025$.

[31] M. Mateo, E. W. Olszewski, and H. L. Morrison, ApJL 508, L55 (1998), arXiv:astro-ph/9810015.

[32] M. Bellazzini, R. A. Ibata, S. C. Chapman, A. D. Mackey, L. Monaco, M. J. Irwin, N. F. Martin, G. F. Lewis, and E. Dalessandro, AJ 136, 1147 (2008), 0807.0105.

[33] S. R. Majewski, M. F. Skrutskie, M. D. Weinberg, and J. C. Ostheimer, ApJ 599, 1082 (2003), arXiv:astro$\mathrm{ph} / 0304198$.

[34] D. R. Law, K. V. Johnston, and S. R. Majewski, ApJ 619, 807 (2005), arXiv:astro-ph/0407566.

[35] D. R. Law, S. R. Majewski, M. F. Skrutskie, and K. V. Johnston, in Satellites and Tidal Streams, edited by F. Prada, D. Martinez Delgado, and T. J. Mahoney (2004), vol. 327 of Astronomical Society of the Pacific Conference Series, pp. 239-+.

[36] J. Peñarrubia, J. F. Navarro, A. W. McConnachie, and N. F. Martin, ApJ 698, 222 (2009), 0811.1579.

[37] B. Moore, S. Ghigna, F. Governato, G. Lake, T. Quinn, J. Stadel, and P. Tozzi, ApJL 524, L19 (1999), arXiv:astro-ph/9907411.

[38] A. Klypin, A. V. Kravtsov, O. Valenzuela, and F. Prada, ApJ 522, 82 (1999), arXiv:astro-ph/9901240.

[39] J. D. Simon and M. Geha, ApJ 670, 313 (2007), 0706.0516 .

[40] M. L. Mateo, ARA\&A 36, 435 (1998), arXiv:astro$\mathrm{ph} / 9810070$.

[41] M. Kesden, ArXiv e-prints (2009), 0903.4458.

[42] I.-G. Jiang and J. Binney, MNRAS 314, 468 (2000), arXiv:astro-ph/9908025.

[43] S. Kazantzidis, L. Mayer, C. Mastropietro, J. Diemand, J. Stadel, and B. Moore, ApJ 608, 663 (2004), arXiv:astro-ph/0312194.

[44] A. Nusser, S. S. Gubser, and P. J. Peebles, Phys. Rev. D 71, 083505 (2005), arXiv:astro-ph/0412586.

[45] S. S. McGaugh, in IAU Symposium, edited by J. Davies and M. Disney (2008), vol. 244 of IAU Symposium, pp. 136-145.

[46] A. S. Eddington, MNRAS 76, 572 (1916).
[47] C. Power, J. F. Navarro, A. Jenkins, C. S. Frenk, S. D. M. White, V. Springel, J. Stadel, and T. Quinn, MNRAS 338, 14 (2003), arXiv:astro-ph/0201544.

\section{APPENDIX A: SATELLITE CONSTRUCTION}

We follow the recipe in [18], with minor adjustment to accommodate two components, stars embedded in a more massive DM halo. In this method, the single particle distribution function $f$ in phase space is a function only of the dynamical constant $Q$,

$$
Q \equiv \varepsilon-\frac{L^{2}}{2 r_{a}^{2}},
$$

where $\varepsilon$ is the total binding energy per unit mass, $\mathbf{L}$ is the particle angular momentum vector per unit mass, and $r_{a}$ is the scale of anisotropy, given as

$$
1-\frac{\sigma_{\theta}^{2}}{\sigma_{r}{ }^{2}}=\frac{r^{2}}{r^{2}+r_{a}^{2}} .
$$

The distribution function gives the number density,

$$
\rho(r)=\int f(\varepsilon, L) d^{3} v .
$$

The inverse of this equation is [46]

$$
f(Q)=\frac{1}{\sqrt{8 \pi}}\left[\int \frac{d^{2} \rho_{Q}}{d \psi^{2}}+\frac{1}{\sqrt{Q}}\left(\frac{d \rho_{Q}}{d_{\psi}}\right)_{\psi=0}\right],
$$

where $\rho_{Q} \equiv \rho(r)\left(1+r^{2} / r_{a}{ }^{2}\right)$ and $\psi \equiv-\Phi-\Phi_{s}$ is the relative potential, including its gravitational and scalar components.

We construct two distribution functions for the DM and the stars in a satellite. The relative potential of the stars is

$$
\Psi_{b}=\int_{r}^{\infty} \frac{G}{r^{2}}\left[M_{\mathrm{b}}(r)+M_{\mathrm{dm}}(r)\right] d r,
$$

where $M_{\mathrm{b}}(r)$ and $M_{\mathrm{dm}}(r)$ are the masses in stars and DM within radius $r$. The relative potential of the DM is

$$
\Psi_{d m}=\int_{r}^{\infty} \frac{G}{r^{2}}\left[M_{\mathrm{b}}(r)+(1+\beta) M_{\mathrm{dm}}(r)\right] d r .
$$

Convergence criteria [47] for numerical sampling of the mass and velocity distribution functions require that the number of particles within the virial radius satisfies

$$
N_{\text {vir }}>\frac{\left(2 c_{\mathrm{v}}\right)^{4}}{\left(\ln \left(1+c_{\mathrm{v}}\right)-c_{\mathrm{v}} /\left(1+c_{\mathrm{v}}\right)\right)^{2}}
$$

for an NFW profile with concentration $c_{\mathrm{v}}$, and the smoothing length $\epsilon$ is

$$
\epsilon=4 \frac{r_{\mathrm{vir}}}{\sqrt{N_{\mathrm{vir}}}} .
$$


Under these conditions there are enough particles that the relaxation time is much longer than the simulation dynamical time, and particle accelerations do not exceed the limit set by the smoothing length that keeps the acceleration induced by two-particle interactions weaker than the mean field halo acceleration.

Our numerical satellite construction code applied to a Hernquist potential [13] yields a distribution function consistent with the analytic solution to better than a part in $10^{5}$ except at the edges of the distribution. We find similar consistency for the combined NFW and plummer profiles, where we numerically constructed the distribution functions from the analytic profiles and then numerically reconstructed from the distribution functions the density runs of the two components. 